\title{
Lanosterol synthase mutations cause cholesterol deficiency-associated cataracts in the Shumiya cataract rat
}

\author{
Masayuki Mori, ${ }^{1}$ Guixin Li, ${ }^{1}$ Ikuro Abe, ${ }^{2}$ Jun Nakayama, ${ }^{3}$ Zhanjun Guo,, ${ }^{1}$ Jinko Sawashita, ${ }^{1}$ \\ Tohru Ugawa, ${ }^{4}$ Shoko Nishizono, ${ }^{5}$ Tadao Serikawa, ${ }^{6}$ Keiichi Higuchi, ${ }^{1}$ and Seigo Shumiya ${ }^{7}$ \\ 1Department of Aging Biology, Institute on Aging and Adaptation, Shinshu University Graduate School of Medicine, Matsumoto, Japan. \\ ${ }^{2}$ School of Pharmaceutical Sciences, University of Shizuoka, Shizuoka, Japan. ${ }^{3}$ Department of Pathology, Shinshu University School of Medicine, \\ Matsumoto, Japan. ${ }^{4}$ Cardiovascular Diseases Research, Yamanouchi Pharmaceutical Co. Ltd., Tokyo, Japan. ${ }^{5}$ Department of Nutrition, \\ Faculty of Nursing and Nutrition, Siebold University of Nagasaki, Nagasaki, Japan. ${ }^{6}$ Institute of Laboratory Animals, Graduate School of Medicine, \\ Kyoto University, Kyoto, Japan. ${ }^{7}$ Department of Laboratory Animal Science, Tokyo Metropolitan Institute of Gerontology, Tokyo, Japan.
}

The Shumiya cataract rat (SCR) is a hereditary cataractous strain. It is thought that the continuous occurrence of poorly differentiated epithelial cells at the bow area of the lens forms the pathophysiological basis for cataract formation in SCRs. In this study, we attempted to identify the genes associated with cataract formation in SCRs by positional cloning. Genetic linkage analysis revealed the presence of a major cataract locus on chromosome 20 as well as a locus on chromosome 15 that partially suppressed cataract onset. Hypomorphic mutations were identified in genes for lanosterol synthase (Lss) on chromosome 20 and farnesyl diphosphate farnesyl transferase 1 (Fdft1) on chromosome 15, both of which function in the cholesterol biosynthesis pathway. A null mutation for Lss was also identified. Cataract onset was associated with the specific combination of Lss and Fdft1 mutant alleles that decreased cholesterol levels in cataractous lenses to about $57 \%$ of normal. Thus, cholesterol insufficiency may underlie the deficient proliferation of lens epithelial cells in SCRs, which results in the loss of homeostatic epithelial cell control of the underlying fiber cells and eventually leads to cataractogenesis. These findings may have some relevance to other types of cataracts, inborn defects of cholesterol synthesis, and the effects of cholesterol-lowering medication.

\section{Introduction}

Cataracts, a leading cause of blindness worldwide, represent a multifactorial eye disease. No method apart from surgical intervention has been shown to be effective in halting cataract formation in the lens. Distinct types of cataracts can be defined according to the section of the lens that becomes opaque and the time of onset. Several risk factors for cataract formation have been identified, including increasing age, genetic predisposition, oxidative stress, exposure to UV light and other toxic agents, and diseased conditions such as diabetes.

Animal models have greatly contributed to the study of cataracts (reviewed in ref. 1). The Shumiya cataract rat (SCR) is a hereditary cataractous rat strain (2). SCRs develop mature cataracts at around 11 weeks of age, exhibiting opacity from the perinuclear zone to the cortical intermediate layer (2-4). The morphological mechanism that causes cataract onset is the extension of anterior sutural hypoplasia and liquefied anterior cortical fibers toward the posterior subcapsular region $(3,4)$. Mild dysplasia of the anterior suture, aggregated epithelial cells overlying the sutural area, and poorly differentiated epithelial cells at the bow area are observable in SCRs from 2 weeks of age (3). The vertebrate eye lens contains only a single layer of epithelial cells on its anterior surface. Lens epithelial cells are essential for maintaining the

Nonstandard abbreviations used: FDFT1, farnesyl diphosphate farnesyl transferase 1; LSS, lanosterol synthase; SCR, Shumiya cataract rat; U18666A, 3- $\beta$ (2-diethylaminoethoxy)-androst-5-en-17-one hydrochloride.

Conflict of interest: The authors have declared that no conflict of interest exists. Citation for this article: J. Clin. Invest. 116:395-404 (2006). doi:10.1172/JCI20797. metabolic homeostasis and transparency of the entire lens, such that it is thought that the continuous occurrence of poorly differentiated epithelial cells at the bow area is responsible for cataract formation in the SCR lens (3). Intriguingly, the lens epithelium is also the initiation site for other types of cataracts, such as selenite-induced cataracts in rabbits (5), UV cataracts in cultured rat lenses (6), rat sugar cataracts (7), and, more importantly, noncongenital cataracts in humans (8).

It is well known that during the development of a mature cataract, degeneration and liquefaction of the remaining lens fibers progresses until the lens becomes liquefied, emulsified, membranous, and finally flattened, leading to a hypermature cataract. However, in the SCR model, following the development of mature cataracts at around 11 weeks of age, normal cortical fibers are regenerated. The lens recovers at around 12 months of age, leaving opacity only in the perinuclear zone (4). This implies that the SCR model may be useful for studies of cataract therapy or even prevention.

The biochemical processes leading to lens opacity have been extensively studied in SCRs, which has revealed upregulation of inducible nitric oxide synthase and elevated calcium influx into lens cells $(9)$, calpain activation $(10,11)$, and enhancement of proteolysis of crystallins and cytoskeletal proteins (12). Another unique feature in SCRs is the hereditary nature of the condition. Although SCR is an inbred strain, the mean cataract occurrence in progeny of cataractous SCR sib-pairs is about two-thirds, while one-third of progeny remains normal. Necropsy at 21 days after mating reveals that about $25 \%$ of the embryos die in utero, so it appears that 1 copy of a recessive gene associated with embryonic 


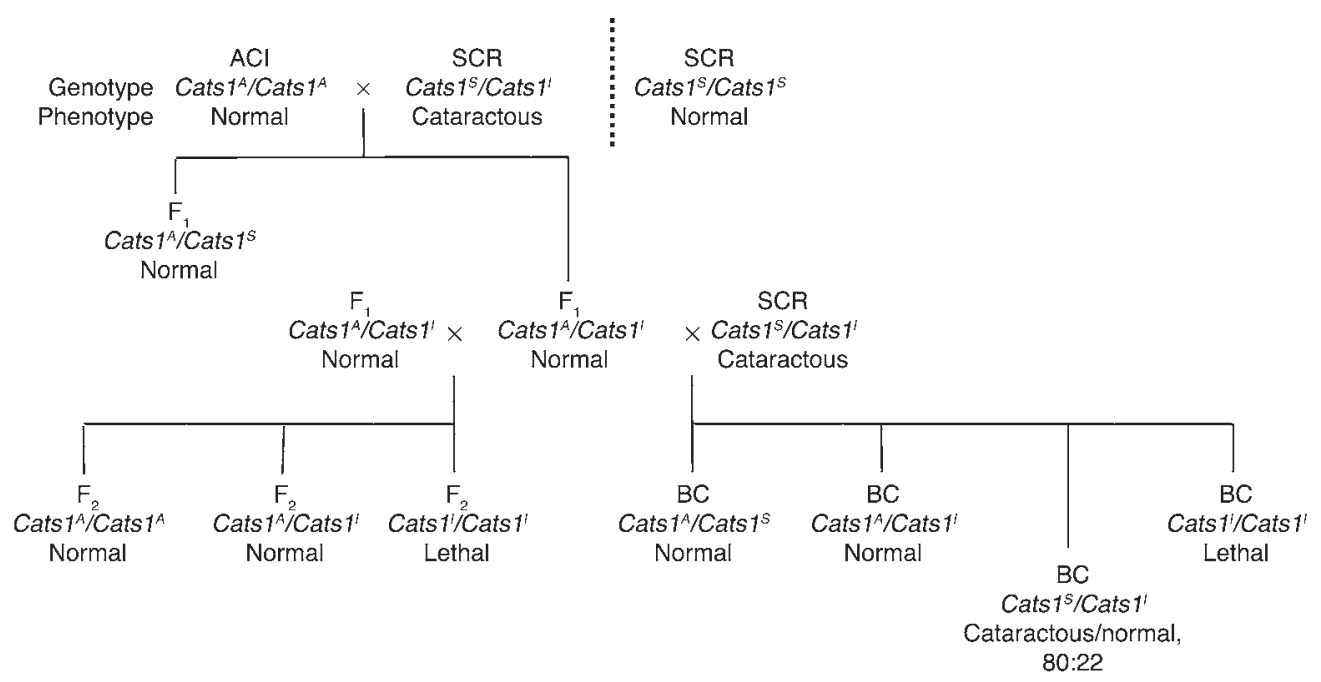

\section{Figure 1}

Graphic representation of the rat mating scheme. The test cross to distinguish $F_{1}$ rats with and without Cats ${ }^{1}$ is not shown. Putative genotypes were assigned to rats with normal, cataractous, or lethal phenotypes. BC, backcross. lethality is a prerequisite for cataract onset in SCRs. While subsequent crossing experiments have revealed a second recessive locus essential for cataract onset (2), it remains unclear how the 2 loci interact. Thus, even though the SCR model has been well characterized mechanistically, the primary genetic defect underlying cataractogenesis has not yet been determined. Elucidation of the function of cataract-associated genes would help establish the sequence of biochemical, cellular, developmental, and physiological events that lead from lens epithelial cell damage to cataract formation. To identify such genes, we used positional cloning in the SCR model. Here we show that cataract onset was associated with the specific combination of mutant alleles of lanosterol synthase (Lss) and farnesyl diphosphate farnesyl transferase 1 (Fdft1) genes, both of which function in the cholesterol biosynthesis pathway. Thus, the SCR model represents a new example of hereditary cholesterol deficiency-associated cataracts. Furthermore our study may have implications for 2 known clinical settings: patients with malformation syndrome due to inborn defects of cholesterol synthesis and individuals taking cholesterol-lowering medication.

\section{Results}

A major gene for cataract onset is located on chromosome 20. We performed chromosomal mapping of cataract genes using $(\mathrm{SCR} \times \mathrm{ACI})$ $\times \mathrm{SCR}$ backcrosses and $(\mathrm{SCR} \times \mathrm{ACI}) \mathrm{F}_{2}$ progenies (Figure 1$)$. Eighty of 305 backcross rats developed cataracts by 12 weeks of age. These cataractous rats were genotyped for microsatellite markers. Marker loci on chromosomes 15 and 20 showed significant deviation toward homozygosity for the SCR allele (Figure 2, A and B).

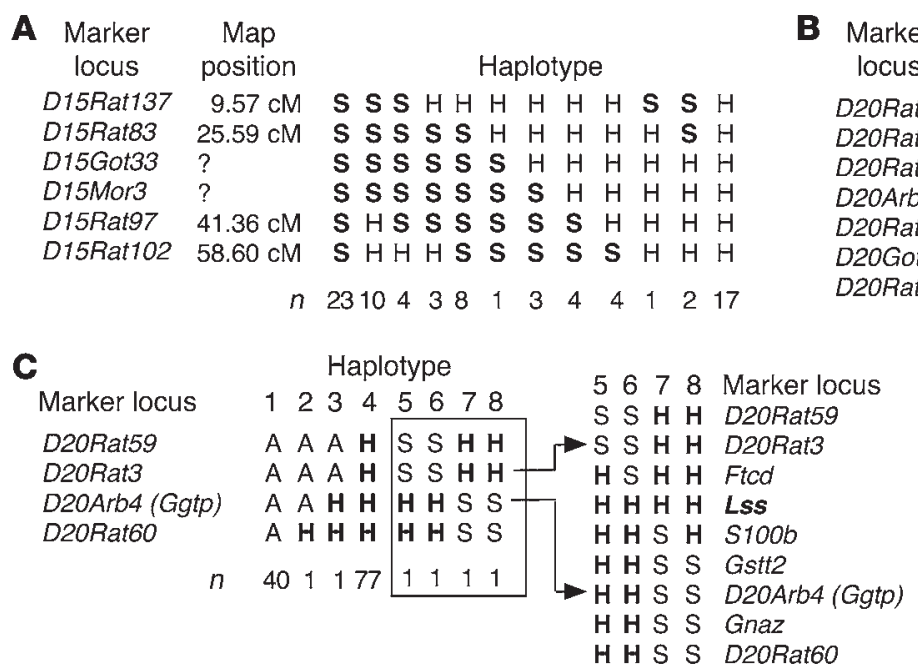

$\begin{array}{lll}\begin{array}{l}\text { Marker } \\ \text { locus }\end{array} & \begin{array}{c}\text { Map } \\ \text { position }\end{array} & \begin{array}{c}\text { Haplotype } \\ \text { D20Rat48 }\end{array} \\ \text { D20Rat59 } & 4.68 \mathrm{cM} & \text { S H S S S S S S S S } \\ \text { D20Rat3 } & 4.68 \mathrm{cM} & \text { S S S S S S } \\ \text { D20Arb4 (Ggtp) } & 6.90 \mathrm{cM} & \text { S S H S S } \\ \text { D20Rat60 } & 6.90 \mathrm{cM} & \text { S S S H S S } \\ \text { D20Got7 } & 9.10 \mathrm{cM} & \text { S S S H H S } \\ \text { D20Rat5 } & & \text { S S S H H H H } \\ & n & 70211132\end{array}$

D

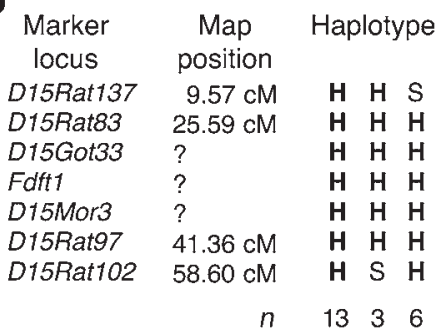

Figure 2

Distribution of haplotypes for chromosomes 15 (A) and 20 (B) observed among 80 cataractous rats from the backcross of (cataractous SCR $\times$ ACl) $F_{1}$ (putative genotype Cats $1^{A} / C$ ats $\left.1^{\prime}\right) \times$ cataractous SCR. (C) Distribution of chromosome 20 haplotypes observed among the 123 noncataractous $F_{2}$ rats from the cross of (cataractous $\mathrm{SCR} \times \mathrm{ACl}) \mathrm{F}_{1}$ (putative genotype Cats $\left.1^{\mathrm{A}} / \mathrm{Cats} 1^{\prime}\right)$. The 4 rats homozygous for SCR-derived alleles (haplotypes 5-8) are boxed. A more detailed haplotype of the 4 rats is shown on the right. (D) Distribution of haplotypes for chromosome 15 among the 22 normal backcross rats with the $L s s^{s} / L s s^{\prime}$ genotype. S, $\mathrm{H}$, and A represent homozygosity for the SCR allele, heterozygosity for the SCR and ACI alleles, and homozygosity for the $\mathrm{ACl}$ allele, respectively. 
A

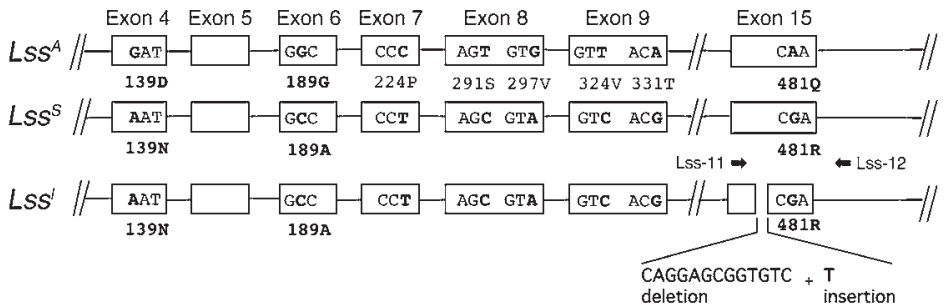

B

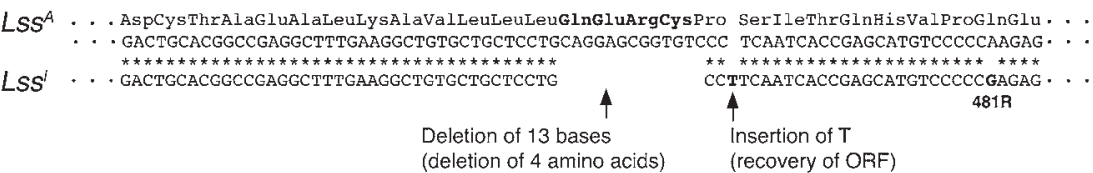

\section{Figure 3}

Summary of mutations observed in the rat $L s S$ gene. (A) Comparison of $L s s^{A}, L s s^{S}$, and $L s s^{\prime}$ chromosomal alleles. Eight exonic nucleotide substitutions and the 3 amino acid substitutions are indicated in bold. Approximate positions of primers Lss-11 and Lss-12 used for genotyping are also shown. (B) Partial nucleotide sequence of exon 15 of the rat $L s s$ gene. The nucleotide sequence of the mutant $L s s^{\prime}$ allele is aligned with that of the normal $L s s^{A}$ allele, with nucleotide matches indicated by asterisks. The Q481R substitution shared with the $L s s^{\prime}$ and $L s s^{S}$ alleles, and the $4-$ amino acid deletion H469-C472del are indicated in bold.
On chromosome 15 , the largest deviation was observed at D15Mor3 (homozygosity/heterozygosity, 52:28; $\chi^{2}=7.2 ; P<0.05$ ). An even larger deviation was observed at D20Rat3 on chromosome 20 (homozygosity/heterozygosity, 80:0; $\chi^{2}=80.0 ; P<0.001$ ), which suggested the nearby presence of a major cataract gene (tentatively designated Cats1 for cataract Shumiya 1). Haplotype analysis assigned the Cats 1 locus to the region between D20Rat59 and D20Arb4, a microsatellite within the Ggtp locus.

Genes for cataract and embryonic lethality are allelic. Intriguingly, none of the $123(\mathrm{SCR} \times \mathrm{ACI}) \mathrm{F}_{2}$ rats developed cataracts by 12 weeks of age. As the backcross data indicated a critical Cats1 region on chromosome 20 , the $\mathrm{F}_{2}$ rats were genotyped for marker loci on this chromosome. Haplotype analysis indicated that $119 \mathrm{~F}_{2}$ rats (with haplotypes 1-4; Figure 2C) were clearly homozygous or heterozygous for ACI-derived alleles. Two rats each were homozygous for SCR-derived alleles at D20Rat3 and Ggtp (D20Arb4). However, the 2 rats homozygous at D20Rat3 (haplotypes 5 and 6) were heterozygous at Ggtp, and the 2 rats homozygous at Ggtp (haplotypes 7 and 8) were heterozygous at D20Rat3. It was probable that these 4 rats had lost homozygosity for the region between D20Rat3 and Ggtp due to recombination in the interval between the loci. Thus, no $\mathrm{F}_{2}$ rat was obtained that was homozygous for SCRderived alleles between D20Rat3 and Ggtp. Prenatal death caused by homozygosity for an SCR-derived allele within this region may

A

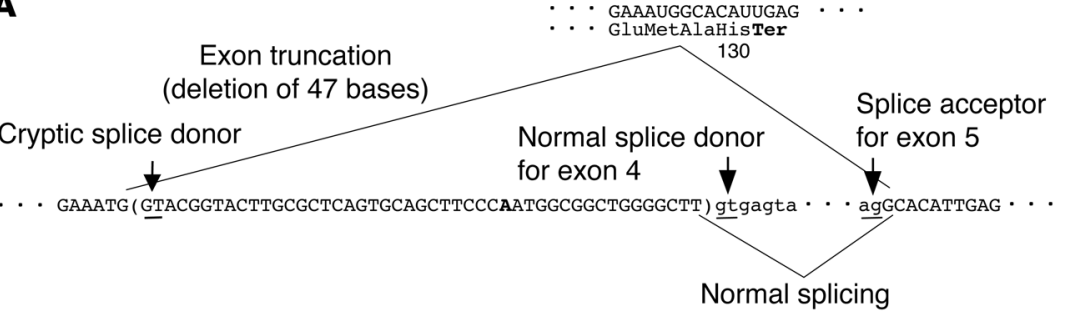

\section{Figure 4}

Exon truncation due to a $G \rightarrow A$ substitution in exon 4 of the rat Lss gene. (A) Exon and intron sequences are indicated by upper- and lower-case letters, respectively. The A substitution in exon 4 is indicated in bold. The 47 bases deleted by the exon truncation are in parentheses. Also shown are the normal (bottom) and aberrant (top) mRNA sequences and the predicted amino acids. The asparagine substitution at residue 139 is indicated in bold. (B) Agarose gel image of RT-PCR products for $L s s$ mRNA derived from the livers and lenses of SCR and $\mathrm{ACl}$ rats as well as minigene constructs. (C) Measurement of aberrant splicing by in vitro transcription of rat Lss minigene constructs. Left: Schematic illustrations of the Lss minigene constructs. Only inserts in the pEF6/V5-His-TOPO vector are shown. Exons, introns, and primers are not to scale. Arrows indicate oligonucleotide primers. Right: Electropherograms of RT-PCR products from each construct. Peak area values and percentages of total are shown for aberrant and normally spliced products.
. GAAAUGGUACGGUACUUGCGCUCAGUGCAGCUUCCCAAUGGCGGCUGGGGCUUGCACAUUGAG * . . - GluMetValArgSerLeuArgSerValglnLeuProAsnGlyGlyTrpGlyLeuHisI leGlu . .

B ACI SCR SCR SCR Lss ${ }^{4}-L_{\text {LS }}$. lens lens liver liver exon $4 / 5$ exon $4 / 5$

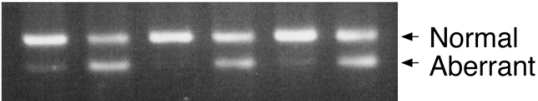

C

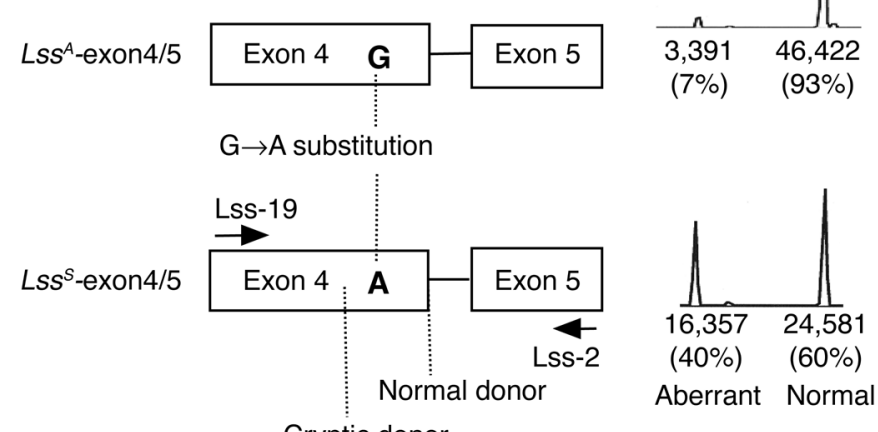

Cryptic donor 


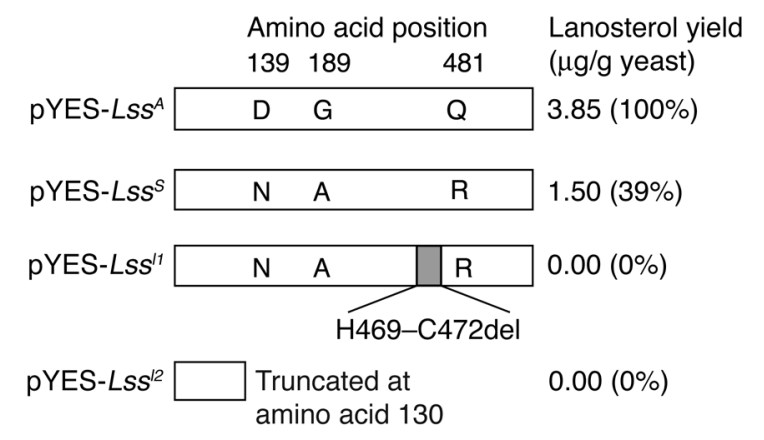

\begin{tabular}{|c|c|c|c|c|}
\hline pYES-LSS ${ }^{A-139 N}$ & $\mathbf{N}$ & $G$ & $\mathrm{Q}$ & $1.23(32 \%)$ \\
\hline pYES-LSS $S^{A-189 A}$ & $D$ & $\mathbf{A}$ & $Q$ & $4.95(110 \%)$ \\
\hline pYES-LSS $S^{A-481 R}$ & D & $\mathrm{G}$ & $\mathbf{R}$ & $3.23(85 \%)$ \\
\hline pYES-Lss ${ }^{S-139 D}$ & D & A & $\mathrm{R}$ & $4.00(104 \%)$ \\
\hline pYES- $L s S^{S-189 G}$ & $\mathrm{~N}$ & $\mathbf{G}$ & $\mathrm{R}$ & $1.89(49 \%)$ \\
\hline pYES-LsS ${ }^{s-481 Q}$ & $\mathrm{~N}$ & A & $\mathbf{Q}$ & $1.19(31 \%)$ \\
\hline
\end{tabular}

\section{Figure 5}

In vitro LSS activity measured as lanosterol yield in yeast. Amino acid variations of the isoforms expressed from each pYES construct are indicated by bold letters. The percentages in parentheses are calculated relative activity when the activity obtained from $\mathrm{pYES}-L s s^{A}$ was defined as $100 \%$.

underlie this observation. Indeed, it has previously been postulated that a recessive cataract gene and a gene associated with embryonic lethality are required for cataract onset in SCRs (2). Taken together, our results strongly suggest that genes for cataract and embryonic lethality are alleles on the same Cats1 locus, Cats $1^{S}$ and Cats $1^{l}$ (with $S$ and 1 referring to SCR and lethal alleles, respectively). Therefore, the genotypes of cataractous and normal SCRs were assumed as Cats $1^{S} /$ Cats $^{l}{ }^{l}$ and $C a t s 1^{S} /$ Cats $^{S}$, respectively (Figure 1). Rats with the Cats $1^{l} /$ Cats $^{l}{ }^{l}$ genotype would die in utero. The ACI strain must carry a dominant Cats $1^{A}$ allele. Accordingly, the $(\mathrm{SCR} \times \mathrm{ACI}) \mathrm{F}_{1}$ rats used for the production of $\mathrm{F}_{2}$ progeny would have a Cats $1^{\mathrm{A}} / \mathrm{Cats}^{l}$ genotype, with the genotype of live-born $(\mathrm{SCR} \times \mathrm{ACI}) \mathrm{F}_{2}$ rats being either $\operatorname{Cats} 1^{A} / \operatorname{Cats}^{A}$ or $\operatorname{Cats}^{A} / \mathrm{Cats}^{l}$, and all rats displaying a normal phenotype. The absence of cataracts in $\mathrm{F}_{1}$ and $\mathrm{F}_{2}$ rats with the Cats $1^{A} /$ Cats $1^{l}$ genotype would then be explained by assuming the order of dominance as follows: $\operatorname{Cats}^{A}, \operatorname{Cats}^{S}$, and Cats $1^{l}$.

Lss is a positional candidate for Cats1. If the above supposition is true, then the $4 \mathrm{~F}_{2}$ rats with haplotypes 5-8 would be Cats $1^{A} /$ Cats $^{l}$ heterozygotes. Therefore, we attempted to narrow down the region for Cats 1 by looking for loci where all 4 of the $\mathrm{F}_{2}$ rats were heterozygous. The Ggtp locus is located near several genes including Gnaz, Gstt2, S100b, Ftcd, and Lss on mouse chromosome 10. Given syntenic conservation between rat chromosome 20 and mouse chromosome 10, we developed microsatellite markers within or close to the rat Gnaz, Gstt2, S100b, and Ftcd genes and genotyped the $4 \mathrm{~F}_{2}$ rats for these markers. At least 1 of the rats was homozygous for SCR-derived alleles at these genes (Figure 2C), which meant none of these genes could be Cats1. For the remaining gene, Lss, we identified a single nucleotide polymorphism (SNP) in PCR products derived from ACI rats and SCRs (data not shown). All 4 rats were heterozygous for the Lss SNP, which suggested that Lss is in fact Cats 1 and that SCRs contain a genetic defect within the Lss locus. Also in support of this hypothesis is the fact that Lss codes for lanosterol synthase (also known as oxidosqualene cyclase; EC 5.4.99.7), which catalyzes the conversion of (3S)-2,3-oxidosqualene to lanosterol, and oral administration of LSS inhibitors is known to induce cataracts in rats (13-16), dogs, and mice (17).

The Lss gene is mutated in SCRs. We compared the nucleotide sequences of the Lss CDNA from ACI rats, cataractous SCRs, and normal SCR littermates. Two different cDNAs were obtained from normal SCRs (presumably with the $L s s^{S} / L s s^{S}$ genotype). One sequence represented the same 2,202-bp open reading frame as the ACI-derived allele $\left(L s s^{A}\right)$ except for 8 nucleotide substitutions, of which 5 were silent mutations $(672 \mathrm{C} \rightarrow \mathrm{T}, 873 \mathrm{~T} \rightarrow \mathrm{C}, 891 \mathrm{G} \rightarrow \mathrm{A}$, $972 \mathrm{~T} \rightarrow \mathrm{C}$, and $993 \mathrm{~A} \rightarrow \mathrm{G})$. The remaining 3 nucleotide substitutions gave rise to missense mutations: $415 \mathrm{G} \rightarrow \mathrm{A}(\mathrm{D} 139 \mathrm{~N})$, $566 \mathrm{G} \rightarrow \mathrm{C}(\mathrm{G} 189 \mathrm{~A})$, and $1442 \mathrm{~A} \rightarrow \mathrm{G}(\mathrm{Q} 481 \mathrm{R})$ (Figure $3 \mathrm{~A})$. The other cDNA sequence from normal SCRs contained the same 8 substitutions as well as a 47-bp deletion (385-431del) that resulted in a reading frame shift and a protein truncated at amino acid position 130. This deletion was later found to be the result of alternative splicing from the $L s s^{S}$ allele.

Four different cDNA sequences were obtained from cataractous SCRs (presumably having the $L s s^{S} / L s s^{l}$ genotype). While 2 were identical to those obtained from normal SCRs (and therefore probably derived from the $L s s^{S}$ allele), the third and fourth cDNA sequences contained a 13-bp deletion (1405-1417del) as well as a single nucleotide insertion (1419-1420insT) in the first and second types, respectively. The 1405-1417del plus the 1419-1420insT led to the loss of 4 amino acids in the LSS protein (H469-C472del) (Figure 3B). The third and fourth cDNA sequences were later found to be alternative splicing products from the $L s s^{l}$ allele. Thus, 3 alleles $\left(L s s^{A}, L s s^{S}\right.$, and $\left.L s s^{l}\right)$ were identified at the Lss locus, which was consistent with the presumption of 3 Cats 1 alleles and further supported the hypothesis that Lss and Cats 1 were the same locus.

Nucleotides 1405-1419 in Lss cDNA corresponded to positions 85 to 101 of exon 15 of the Lss gene. When we PCR amplified and sequenced Lss exon 15 from cataractous SCR, 2 different sequences were obtained: 1 with and 1 without the mutations corresponding to $1405-1417 \mathrm{del}$ and $1419-1420 \mathrm{ins}$. Therefore, the $1405-1417 \mathrm{del}$ and 1419-1420insT alterations reflected genomic mutations.

Aberrant splicing of Lss $m R N A$ is due to a single nucleotide substitution in exon 4. The 385-431del in Lss cDNA corresponded to the last 47 nucleotides (position 63 to 109) of exon 4 of the Lss gene. Examination of exon 4 sequences from normal and cataractous SCRs revealed that the 47 nucleotides missing from the mRNA

\section{Table 1}

Segregation at $D 15 M o r 3$ in $(\mathrm{SCR} \times \mathrm{ACI}) \times \mathrm{SCR}$ backcross rats with the $L s S^{S} / L s S^{\prime}$ genotype

\begin{tabular}{lccc} 
Genotype at & \multicolumn{3}{c}{ Phenotype } \\
D15Mor3 & Cataractous & Normal & Total \\
S/S & 52 & 0 & 52 \\
S/A & 28 & 22 & 50 \\
Total & 80 & 22 & 102 \\
\hline
\end{tabular}


A

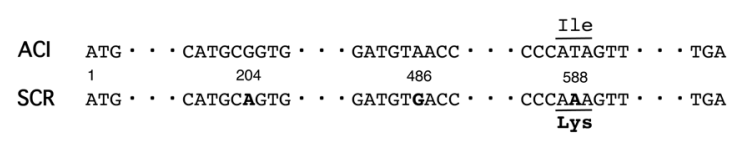

B
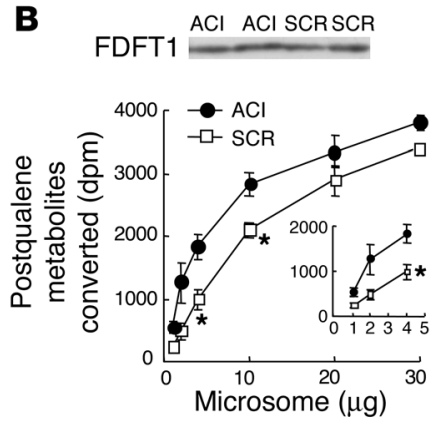

Figure 6

Fdft1 mutations and reduced FDFT1 activity in SCRs. (A) Alignment of regions of Fdft1 cDNA sequence derived from SCR and ACl rats. Nucleotide substitutions at positions 204, 486, and 588 in SCR Fdft 1 are indicated in bold. The I196K amino acid substitution caused by $588 \mathrm{~T} \rightarrow \mathrm{A}$ is also shown. (B) Comparison of FDFT1 activity in rat liver microsome, as determined by amount of postsqualene metabolites converted from $\left[{ }^{3} \mathrm{H}\right]$ farnesyl diphosphate (mean dpm $\pm \mathrm{SD}$ ). ${ }^{*} P<0.05$ versus $\mathrm{ACl}$ rats (Student's $t$ test). Western blot image of rat hepatic FDFT1 is shown at the top.

were still present in genomic DNA. Indeed, only 1 difference was observed between the 2 Lss alleles, as position 93 in exon 4 contained a $\mathrm{G}$ in $L s s^{A}$ but an $\mathrm{A}$ in $L s s^{S}$, which corresponds to the $415 \mathrm{G} \rightarrow \mathrm{A}$ substitution in Lss cDNA. Based on this finding, we hypothesized that the 385-431del in Lss cDNA was due to aberrant mRNA splicing that utilized the GpT at the $5^{\prime}$ end of $385-$ $431 \mathrm{del}$ as a splice donor (Figure 4A). To confirm this hypothesis, chromosomal DNA spanning from exon 4 to exon 5 of both $L s s^{A}$ and $L s^{S}$ alleles was transcribed in COS cells, and the resultant splicing pattern was examined. Only trace amounts of aberrant transcripts were observed for the $L s s^{A}$ allele (Figure 4, B and C). On the other hand, aberrant transcripts were observed for the $L s s^{S}$ allele, with the aberrant form accounting for about $40 \%$ of total transcripts, which was equivalent to that observed in SCR lenses and livers. These results suggest that the 385431del in Lss cDNA was caused by aberrant splicing at position 72 in exon 4 , and that the $\mathrm{G} \rightarrow \mathrm{A}$ substitution at position 93 in exon 4 was involved in this aberrant splicing. While the mechanisms for this shift in splice donor were unclear, it is possible that the substitution enhanced the affinity of the cryptic splicing site for the splicing apparatus, allowing it to compete with the intrinsic splice site. As an $L s s^{l}$ minigene construct exhibited a splicing pattern similar to that of the $L s s^{S}$ construct (data not shown), our results indicated that the 4 types of mRNA in cataractous SCRs represent normal and aberrant transcripts from $L s s^{S}$ and $L s s^{l}$ alleles.

Mutant LSS isoforms have reduced activity. We then measured LSS activity in the liver. The rate of conversion from oxidosqualene to lanosterol by liver microsomes of cataractous SCRs $\left(L s s^{S} / L s s^{l}\right)$ was $42.9 \pm 4.0 \mathrm{pmol} / \mathrm{mg} / \mathrm{min}$, which was significantly lower than the rate obtained from ACI rats $(113.0 \pm 11.1 \mathrm{pmol} / \mathrm{mg} / \mathrm{min}$; $P<0.001)$, but not different from that obtained from normal SCRs $\left(L s s^{s} / L s s^{s} ; 46.0 \pm 4.5 \mathrm{pmol} / \mathrm{mg} / \mathrm{min}\right)$. The finding that LSS activities were not different between cataractous and normal
SCRs was puzzling. One hypothesis to account for this discrepancy is possible fluctuations in the proportion of aberrantly spliced mRNA. Indeed, we observed differences in the percentages of aberrantly spliced products (range, 31.5-78.8\%; average, $40.5 \%$ ) between rats, although deviations within individual rats were small (data not shown). The lack of difference in LSS activity between normal and cataractous SCRs might be explained if it is assumed that the percentage of aberrant transcripts was increased in normal SCRs or if the percentage of normal transcripts was increased in cataractous SCR used for the LSS activity assay.

To avoid this problem, each LSS isoform was expressed from cDNA in yeast, and LSS activity was assessed (Figure 5). Fulllength LSS with the D139N, G189A, and Q481R amino acid substitutions (full-length form of the $L s s^{S}$ allele) produced only about $39 \%$ of the lanosterol produced by full-length LSS encoded by the $L s s^{A}$ allele. No lanosterol was produced from the H469-C472del Lss ${ }^{l}$ allele, and the LSS truncated at amino acid 130. These results clearly indicate that $L s s^{S}$ and $L s s^{l}$ are hypomorphic and null alleles, respectively. Furthermore, all 3 artificial isoforms carrying the D139N substitution showed reduced lanosterol production, which suggests that the D139N substitution was responsible for the diminished enzyme activity of $L s s^{S}$ allele (Figure 5). Taken together, our results demonstrate that the $\mathrm{G} \rightarrow \mathrm{A}$ nucleotide substitution in exon 4 of the $L s s$ allele leads to reduction of enzyme activity through the D139N amino acid substitution in addition to the activation of a cryptic splice donor.

Cats $2^{A}$ on chromosome 15 suppresses cataractogenesis. We genotyped the Lss locus of 80 cataractous backcross rats and confirmed that all rats were $L s s^{S} / L s s^{l}$ heterozygotes. However, the frequency of cataractous rats was significantly lower than expected ratio of onethird (cataractous/normal, 80:225; $\chi^{2}=7.0 ; P<0.01$ ). To determine whether some of the noncataractous rats carried the $L s s^{S} / L s s^{l}$ heterozygous genotype, we genotyped all 225 normal backcross rats for the Lss locus and found that 22 rats were indeed $L s s^{S} / L s s^{l}$ heterozygotes. Thus, the frequency of rats with the $L s^{S} / L s s^{l}$ genotype was not different from the expected one-third $\left(\chi^{2}=0.1 ; P>0.1\right)$. The absence of cataracts in these $22 \mathrm{Lss}^{S} / \mathrm{Lss}^{l}$ rats could be due to

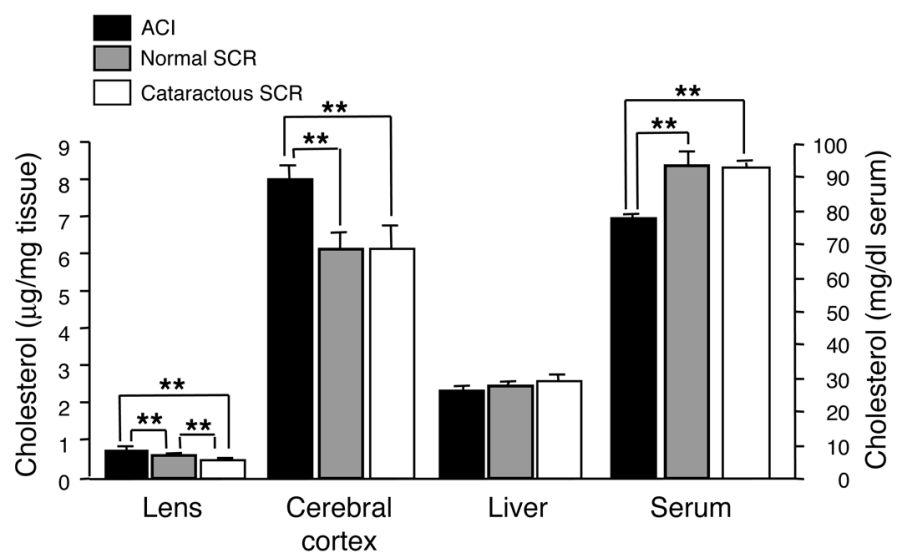

Figure 7

Comparison of tissue and serum cholesterol levels. The mean cholesterol content in $\mathrm{ACl}$ rats, normal SCR, and cataractous SCR ( $n=3$ per group) in the lens $(0.74,0.56$, and $0.42 \mu \mathrm{g} / \mathrm{mg}$ wet tissue, respectively), frontal cerebral cortex $(8.00,6.15$, and $6.15 \mu \mathrm{g} / \mathrm{mg}$ wet tissue, respectively), liver (2.24, 2.42, and $2.56 \mu \mathrm{g} / \mathrm{mg}$ wet tissue, respectively), and serum (77.3, 93.0, and $92.8 \mathrm{mg} / \mathrm{dl}$, respectively) are shown. ${ }^{* \star} P<0.01$. 


\section{Table 2}

Sterol profile in the rat lens

$\begin{array}{lcccccc}\text { Rat strain } & \text { Squalene } & \text { 2,3-oxidosqualene } & \text { Lanosterol } & \text { 7-dehydrocholesterol } & \text { Desmosterol } & \text { Cholesterol } \\ \text { ACl } & 0.014 \pm 0.010 & 0.005 \pm 0.004 & 0.002 \pm 0.001 & 0.005 \pm 0.001 & 0.021 \pm 0.008^{\mathrm{A}} & 0.740 \pm 0.030^{\mathrm{B}} \\ \text { Normal SCR } & 0.019 \pm 0.003 & 0.002 \pm 0.001 & 0.001 \pm 0.000^{\mathrm{C}} & 0.002 \pm 0.000 & 0.032 \pm 0.002^{\mathrm{A}} & 0.560 \pm 0.010^{\mathrm{B}} \\ \text { Cataractous SCR } & 0.020 \pm 0.001 & 0.002 \pm 0.001 & 0.002 \pm 0.000 & 0.001 \pm 0.001 & 0.040 \pm 0.001^{\mathrm{A}} & 0.423 \pm 0.023^{\mathrm{B}}\end{array}$

Values were calculated as $\mu \mathrm{g} / \mathrm{mg}$ lens. ${ }^{\mathrm{A} P}<0.05$, ${ }^{\mathrm{B}} \mathrm{P}<0.01$ between strains; ${ }^{\mathrm{C}} \mathrm{P}<0.05$ compared with $\mathrm{ACl}$.

incomplete penetrance of the effect of the Lss mutation or to intervention by other gene(s) that prevent cataract onset. To investigate these possibilities, the $22 L s s^{S} / L s s^{l}$ rats without cataracts were genotyped for other marker loci. All 22 rats were heterozygous at the region between D15Rat83 and D15Rat97 (homozygosity/heterozygosity, $0: 22 ; \chi^{2}=22.0 ; P<0.001$; Table 1$)$. Meanwhile in cataractous rats, significant deviation toward homozygosity for SCR-derived alleles was observed at these loci (homozygosity/heterozygosity, $\left.52: 28 ; \chi^{2}=7.2 ; P<0.05\right)$. Thus there appeared to be a gene on chromosome 15 of ACI rats that suppressed cataractogenesis (provisionally designated $\left.\operatorname{Cats}^{A}\right)$. The genetic effect of $\mathrm{Cats}^{A}$ appeared to be semidominant, as about $50 \%$ (22 of 50) of the rats with the Cats $2^{S} /$ Cats $2^{A}$ genotype still developed cataracts. Haplotype analysis assigned the Cats2 locus to the region between D15Rat137 (9.57 cM) and D15Rat102 (58.60 cM) (Figure 2D).

SCR has a mutation in the Fdft1 gene and reduced FDFT1 activity. The Cats 2 region seemed too large for positional cloning, but we examined 3 candidate genes for Cats2: gap junction membrane channel protein, alpha 3 (Gja3); Rab geranylgeranyl transferase, a subunit (Rabggta); and Fdft1. These genes were selected because the null mutation of Gja3 is known to lead to nuclear cataracts with crystallin proteolysis (18); RABGGTA shows protein prenylation activity, and the inhibition of prenylation of small GTP-binding proteins is an important factor in the lavastatin-induced rat cataract model (19); and FDFT1 (also known as squalene synthase; EC 2.5.1.21) functions 2 steps upstream of the LSS in the cholesterol biosynthesis pathway, catalyzing the condensation of 2 molecules of farnesyl pyrophosphate to form squalene. cDNA fragments covering the entire coding region of the 3 genes were PCR amplified from the lens mRNA of ACI rats and SCRs, run on an agarose gel, and sequenced. PCR products of Gja3 and Rabggta mRNA derived from the SCR and ACI strains did not show apparent quantitative differences by agarose gel electrophoresis (data not shown). In addition, no strain-specific differences were identified in the cDNA sequences of either gene, which suggested that neither gene was Cats2.

Meanwhile, the sequence of Fdft 1 cDNA revealed 3 nucleotide substitutions between ACI $\left(F d f t 1^{A}\right)$ and SCR $\left(F d f t 1^{S}\right)$. While 2 of these substitutions were silent $(204 \mathrm{G} \rightarrow \mathrm{A}$ and $486 \mathrm{~A} \rightarrow \mathrm{G})$, the third substitution $(588 \mathrm{~T} \rightarrow \mathrm{A})$ was a missense mutation, such that codon 196 was changed from ATA in ACI rats to AAA in SCRs, which resulted in an I196K substitution (Figure 6A). Western blot analysis indicated that roughly equal amounts of FDFT1 were expressed in the livers of SCR and ACI rats (Figure 6B). However, FDFT1 activity in liver microsomal fractions was significantly lower in SCR than in ACI rats, which indicated that the $F d f t 1^{S}$ was a hypomorphic allele. The relatively slight increase in FDFT1 activity in ACI rats appeared to agree well with the semidominant effect of the Cats $2^{A}$ locus. While the results presented in this report are admittedly not sufficient to substantiate the hypothesis that mutated Fdft1 is Cats $2^{S}$, we believe that the $F d f t 1$ is a strong candidate for the Cats2, because in particular FDFT1 functions 2 steps upstream of LSS in the cholesterol biosynthesis pathway. It is at least conceivable that cholesterol biosynthesis was further disturbed by the mutant Fdft1 in SCR, thereby rendering the rats more susceptible to cataracts, and $F d f t 1^{A}$ allele suppressed cataractogenesis by more efficiently catalyzing squalene production.

The SCR lens has low cholesterol content. We then examined lens cholesterol content. Lenses of normal SCRs $\left(L s s^{S} / L s s^{S}\right)$ and cataractous SCRs $\left(L s s^{S} / L s s^{l}\right)$ had cholesterol contents that were $76 \%$

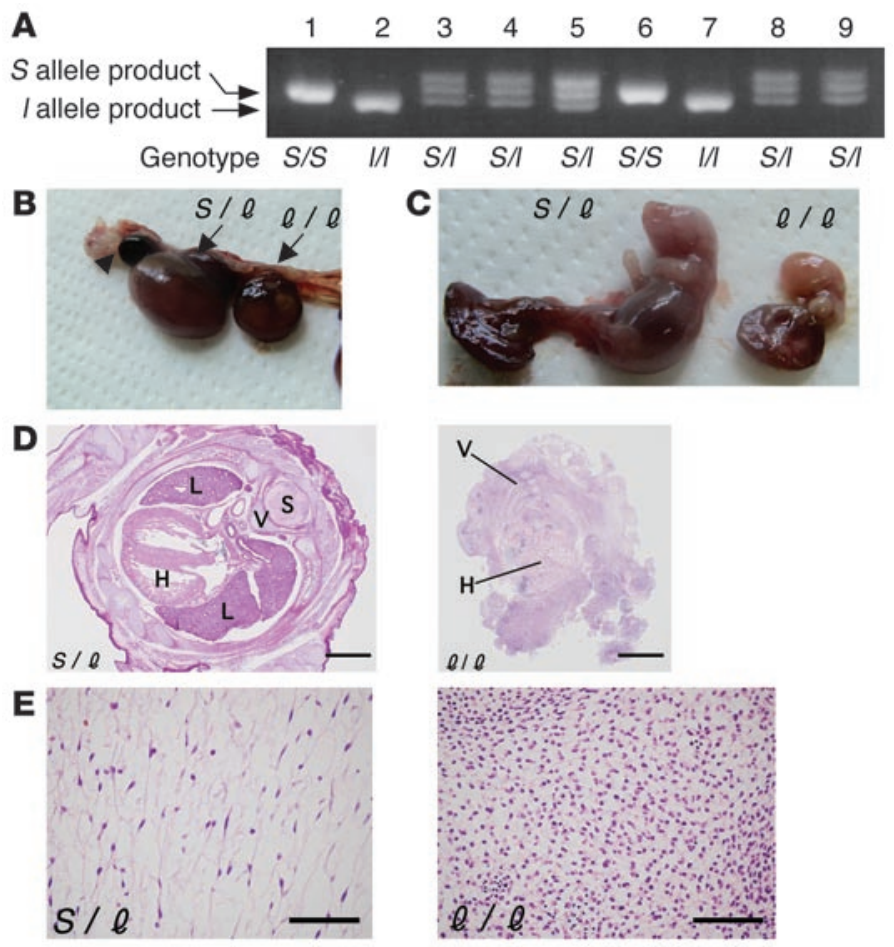

Figure 8

Phenotype of $L s s^{\prime} / L s s^{\prime}$ embryos. (A) Agarose gel electrophoresis image of the genotype analysis for a litter of E12.5 embryos. (B) Gross appearance of part of a uterus with embryos at E16.5. Two embryos covered with the yolk sac are indicated by arrows. One embryo (arrowhead) shows resorption, leaving only placenta. (C) Gross appearance of normal (left) and $L s s^{\prime} / L s s^{\prime}$ embryos (right) shown in $\mathbf{B}$, taken out of the yolk sac with the placenta. (D) Cross sections of the same embryos shown in $\mathbf{C}$. $\mathrm{H}$, heart; $\mathrm{V}$, vertebra; S, spinal cord; L, lung. (E) Higher magnification of mesenchymal tissues. Scale bars: $1 \mathrm{~mm}$ (D); $100 \mu \mathrm{m}$ (E). 
Table 3

Frequency of genotypes in offspring from intercross of $L s S^{S} / L S S^{\prime}$ rats

\begin{tabular}{|c|c|c|c|c|c|c|}
\hline Age & No. litters & $L s s^{S} / L s s^{s}$ & $L s s^{s} / L s s^{\prime}$ & $L s s^{\prime} / L s s^{\prime}$ & Resorbed & Total \\
\hline E9.5 & 2 & 3 & 9 & 4 & 0 & 16 \\
\hline E12.5 & 3 & 7 & 15 & 5 & 0 & 27 \\
\hline E14.5 & 4 & 6 & 14 & 2 & 8 & 30 \\
\hline E16.5 & 3 & 10 & 12 & 3 & 5 & 30 \\
\hline E18.5 & 3 & 8 & 13 & 0 & 7 & 28 \\
\hline Postnatal & 2 & 4 & 10 & 0 & NA & 14 \\
\hline
\end{tabular}

effective beyond midgestation when a functional cholioallantoic circulatory system is established (24). The timing of SCR embryo death appeared to correspond with this period. Nonetheless, a few retarded $L s s^{l} / L s s^{l}$ embryos could still be recovered beyond E16.5 (Figure 8C). The major pathological abnormality in these embryos was defective organogenesis (Figure 8D), with skin and nerve tissues, as well as major internal organs, hardly discernible. The anatomical location of organs was also disturbed. $L s s^{l} / L s s^{l}$ embryos also displayed abnormal mesenchymal tissues (Figure 8E). These mesenchymal cells were small and oval shaped with large nuclei and high cellularity, which was suggestive of immature status. At E18.5, no Lss ${ }^{l} / L_{s s}^{l}$ embryos were identified. Thus, it appeared that the major cause of prenatal death of $L s s^{l} / L s s^{l}$ embryos was dysorganogenesis due to poor cellular proliferation.

\section{Discussion}

Our data identified mutations in the gene for LSS, part of the cholesterol biosynthesis pathway, as the underlying cause of cataracts in the SCR strain and indicated that the SCR represents a new example of hereditary cholesterol deficiency-associated cataracts. Our findings may have implications for 2 known clinical settings: patients with malformation syndromes due to inborn defects of cholesterol synthesis and individuals taking cholesterol-lowering medication. To date, 6 inborn defects of cholesterol biosynthesis have been described in humans (reviewed in refs. $25,26)$, and 3 of the associated conditions have cataracts as complications: mevalonic aciduria (27-29), Smith-Lemli-Opitz syndrome (30-32), and X-linked dominant chondroplasia punctata $2(33,34)$. The cause of cataracts in these diseases was postulated to be due to the decrease in cholesterol level in the lens. Alternatively, the cataracts may reflect a response to the accumulation of precursors in the cholesterol biosynthesis pathway. Our data showing reduced cholesterol level without accumulation of sterol precursors in the SCR lens supports the hypothesis that the common cause of cataracts in these diseases is in fact cholesterol deficiency. Thus the cataracts in these conditions may share developmental features with the SCR model.

The continuous appearance of poorly differentiated epithelial cells at the bow area of the lens is thought to be the pathophysiological basis for cataract formation in the SCR (3). It is thought that the lens derives its cholesterol from de novo synthesis in situ, as vertebrate eye lenses are nonvascularized (35). The vertebrate eye lens contains only a single layer of epithelial cells on its anterior surface. The epithelial cells remain quiescent in the central section of the lens surface, divide toward the equatorial area, and terminally differentiate into fiber cells in the equatorial regions. Cholesterol is required for membrane formation by these proliferating lens epithelial cells to proceed through cell cycle (36). The importance of the epithelial cells is further inferred from the fact that mature lens fiber cells lose their nuclei and other cytosolic organelles during differentiation, and with them the ability to synthesize cholesterol. For these reasons, the lens may be especially vulnerable to the effect of low cholesterol. Based on these observations, we postulated a mechanism for cataract development in SCRs whereby SCR lens epithelial cells unable to synthesize sufficient cholesterol for proper proliferation caused the loss of homeostatic epithelial cell control of the underlying fiber cells, leading to the sequence of biochemical abnormalities observed in 
the SCR, from increased cellular calcium (9) to calpain activation $(10,11)$, enhancement of proteolysis of crystallins and cytoskeletal proteins (12), and eventually protein insolubilization that is responsible for lens opacification.

Intriguingly, it has been pointed out that lens epithelial cell apoptosis could be a common initial cause of noncongenital cataracts (5-8). A typical lens undergoes a series of changes during the cataractous process. First, separation and liquefaction of the anterior suture occur, after which the separated part of the anterior suture gradually becomes covered by proliferative epithelial cells. As a consequence, lens fiber cells regenerate, leading to the recovery of lens transparency. However, upon the completion of repair these proliferative epithelial cells gradually decrease in number and become vacuolated and swollen with age, such that recovery of the cataract is halted. This process shows the importance of the differentiating and metabolizing functions of the epithelial cells in lens reconstruction and raises the possibility that transplantation of normal lens epithelial cells could prevent and/or recover the cataractous lens in SCRs as well as assist in the development of a novel therapeutic and/or prophylactic approaches to human cataracts.

Other abnormal phenotypes observed in human patients with hereditary defects in the cholesterol biosynthesis pathway, such as dysmorphic development, mental retardation, and behavioral phenotype due to neurological defects, are not apparent in SCRs. Compared with ACI rats, the cholesterol content of the frontal cerebral cortex of both normal $\left(L s s^{S} / L s s^{S}\right)$ and cataractous SCRs $\left(L s s^{S} / L s s^{l}\right)$ was reduced by $24 \%$ (Figure 7 ), which still might be sufficient for normal brain development and function. In the liver, LSS activity in SCRs was only about $40 \%$ that of the ACI rats. However, as there were no discernible liver abnormalities in SCR, it appears that the reduced LSS activity was still enough to maintain liver cholesterol levels.

Interfering with cholesterol biosynthesis using drugs is an effective therapeutic approach to lower elevated plasma cholesterol levels and the risks of coronary heart disease. Although these drugs are relatively safe, there are still concerns regarding toxicity. Whether cataract occurrence is increased in patients treated with cholesterol-lowering medications remains uncertain. Of the cholesterollowering agents currently used, statins, which function as potent inhibitors of hydroxymethyl glutaryl coenzyme A (HMG-CoA) reductase, are the most prescribed group of drugs. While results of clinical safety trials collectively show no higher risk of cataracts in statin-treated patients (26), Schlienger et al. (37) reported that concomitant use of erythromycin and simvastatin might increase cataract risk in humans. Erythromycin is thought to block statin metabolism by the cytochrome P-450 system, thereby increasing the serum levels of statins and the risk for toxicity.

Meanwhile, it has been demonstrated in toxicity tests using animals that several drugs that inhibit lens cholesterol biosynthesis can produce cataracts (reviewed in ref. 26). For instance, vastatin causes cataracts in dogs (38). Another group of agents, lanosterol synthase inhibitors, induce cataracts in rats (13-16), mice, and dogs (17). Above all, the cataracts caused by $3-\beta$ (2-diethylaminoethoxy)-androst-5-en-17-one hydrochloride (U18666A) have been intensively studied. Results from the U18666A-induced rat cataract model suggested that total lens cholesterol content needed to be decreased by $30-70 \%$ for cataract onset (13-16). More recent reports suggest that the cataractogenic effect of $\mathrm{U} 18666 \mathrm{~A}$ might be related to direct perturbation of lens membrane structure and function (39). In the SCR model, cataracts occurred only in rats heterozygous for the hypomorphic $L s s^{s}$ and null $L s s^{l}$ alleles, but not in those homozygotes for the $L s s^{S}$ allele. Lenses of cataractous SCRs $\left(L s s^{S} / L s s^{l}\right)$ and normal SCRs $\left(L s s^{S} / L s s^{S}\right)$ had cholesterol contents that were $57 \%$ and $76 \%$ that of ACI lenses, respectively. These results support the hypothesis that cholesterol deficiency alone may induce cataracts, even though the possibility of direct perturbation by U18666A cannot be excluded. Our data also underpinned the hypothesis that there was a distinctive threshold between $57 \%$ and $76 \%$ for lens cholesterol level in cholesterol deficiency-associated cataracts. Threshold cholesterol levels for other organs to maintain normality could not be defined in the SCR model. Defining the threshold should be particularly important, considering that there is no known cure for human syndromes resulting from impaired cholesterol biosynthesis and that cholesterol supplementation is proposed as 1 possible therapy.

Our data also underscored the possibility that even a slight change at a single step in the cholesterol biosynthesis pathway (FDFT1 in the case of the SCR) could compound susceptibility to cholesterol deficiency-associated cataracts when another step (LSS in the case of the SCR) is also restrained. Intriguingly, simvastatin is cataractogenic when administered orally to a specific strain of Wistar rats (40). It was postulated that this rat strain carried a genetic defect in the regulation of a key enzyme downstream of mevalonic acid in the cholesterol biosynthesis pathway that rendered the rats more susceptible to the cataractogenic effects of simvastatin. It is possible that hypomorphic genetic polymorphisms exist in cholesterol biosynthesis genes in humans similar to those seen in rats. The presence of such polymorphisms has been inferred from the observations in Smith-Lemli-Opitz syndrome. These patients have the mutations in the gene for $3 \beta$-bydroxysterol $\Delta^{7}$-reductase (DHCR7), and hence have defects in cholesterol biosynthesis. However, there is a remarkable difference in phenotypic severity even between patients with identical DHCR7 mutations. Thus, it has been postulated that other genetic as well as developmental or maternal factors perhaps affecting cholesterol biosynthesis or homeostasis significantly influence a given patient's phenotype. If in fact hypomorphic genetic polymorphisms exist in cholesterol biosynthesis genes in humans, then it is possible that such people would be at a higher risk for cataracts when receiving statins or other cholesterol-lowering agents. Conversely, exploration of these polymorphisms may shed a new light on the clinical studies of disorders such as Smith-Lemli-Opitz syndrome.

In conclusion, we have identified the molecular genetic basis of cataract onset in the SCR strain. The SCR model represents what we believe to be a new example of hereditary cholesterol deficiency-associated cataracts and should prove useful in the development of therapeutic and/or preventive measures for cataracts.

\section{Methods}

Genetic linkage analysis of cataracts. Normal SCRs, cataractous SCRs, and ACI rats were maintained at the Department of Laboratory Animal Science of the Tokyo Metropolitan Institute of Gerontology. Protocols used in this study were approved by the institute's review board. Cataractous SCRs (putative genotype, $\left.\operatorname{Cats} 1^{S} / \operatorname{Cats}^{l}{ }^{l}\right)$ were crossed to $\mathrm{ACI}$ rats $\left(\operatorname{Cats} 1^{A} / \operatorname{Cats} 1^{A}\right)$ to give $(\mathrm{SCR} \times \mathrm{ACI}) \mathrm{F}_{1}$ rats (Figure 1). To distinguish $\mathrm{F}_{1}$ rats with and without Cats $^{l}$, a prerequisite for cataract onset, rats were crossed with normal SCRs $\left(\operatorname{Cats} 1^{S} / \operatorname{Cats}^{S}\right)$. $F_{1}$ rats that had cataractous progeny were judged as having the Cats $1^{l}$ allele $\left(\operatorname{Cats}^{A}{ }^{A} /\right.$ Cats $\left.^{l}{ }^{l}\right)$. These $\mathrm{F}_{1}$ rats were intercrossed or crossed with cataractous SCRs to give $(\mathrm{SCR} \times \mathrm{ACI}) \mathrm{F}_{2}$ and $(\mathrm{SCR} \times \mathrm{ACI}) \times \mathrm{SCR}$ 
backcross rats. Rats were diagnosed for cataracts at 12 weeks of age by visual inspection and then sacrificed, and liver genomic DNA was extracted. Rats were genotyped for 78 microsatellite marker loci dispersed throughout the genome, and genetic linkage of the markers with cataracts was evaluated by the $\chi^{2}$ test with a $P$ value smaller than 0.05 being accepted as evidence of linkage.

Positional cloning of the cataract gene. Five genes (Gnaz, Gstt2, S100b, Lss, and $F t c d$ ) located centromeric or telomeric to the Ggtp locus were selected from the mouse supercontig data. Rat contigs containing these genes were obtained by BLAST search using the mouse cDNA sequences against the NCBI rat contig databank (http://www.ncbi.nlm.nih.gov/genome/seq/ RnBlast.html). Microsatellites identified in the contigs were examined for polymorphisms between SCRs and ACI rats, with the polymorphic microsatellites then used for the linkage study. Oligonucleotide primers for rat Lss cDNA were designed based on the reported sequence (U31352). Nucleotide sequences of the primers were as follows: Lss-1, 5'-GTGGTCCAGAGCTGTGCTGT-3'; Lss-2, 5'-TGGACTTGTCCTCAATGTGC-3'; Lss-19, 5'-GTCTCCTGATTACGTGTCACATAGC-3'; Lss-4, 5'-TTGATCCTCGAGACGTGCTC-3'; Lss-5, 5'-ATTCGTTGGTCAGTGGACGG-3'; Lss-6, 5'-TTGATCCTCGAGACGTGCTC-3'; Lss-7, 5'-ACAGGGCTACAGAGATCAGG-3'; Lss-8, 5'-GGACGCTTATCACCCAGCTG-3'; Lss-11, 5'-CTTCAGCACACTGGACTGT-3'; Lss-12, 5'-GGCTTTAGACTGATGTCCTAC-3'. Lens mRNA was extracted from SCRs and ACI rats using the QuickPrep Micro mRNA Purification Kit (Amersham Biosciences). First-strand cDNA was synthesized using a First-Strand cDNA Synthesis Kit (Amersham Biosciences), and cDNA fragments containing the entire Lss coding sequence were PCR amplified. An aliquot of each PCR product was then subjected to agarose gel electrophoresis. PCR products were directly sequenced using the primers used for PCR amplification or cloned into the PCR2.1 plasmid vector (Invitrogen Corp.).

LSS activity assay. The LSS activity assay for liver microsomal fractions was performed as previously described (41). Yeast expression constructs were made by cloning cDNA fragments for the 4 LSS isoforms into the pYES2.1 plasmid (Invitrogen Corp.), resulting in pYES-Lss ${ }^{A}$, pYES- $L s s^{S}$, pYES-Lss ${ }^{l 1}$, and PYES- $L s s^{2}$ (Figure 5). Nucleotide substitutions were introduced into the PYES- $L s s^{A}$ and PYES- $L s s^{S}$ using the GeneEditor In Vitro Site-Directed Mutagenesis System (Promega), resulting in pYES-Lss ${ }^{A-139 N}$, pYES$L s s^{A-189 A}$, pYES-Lss ${ }^{A-481 R}$, pYES-Lss ${ }^{S-139 D}$, pYES-Lss ${ }^{S-189 G}$, and pYES-Lss ${ }^{S-4810}$. The LSS-deficient yeast strain, SGL9, was transformed with the constructs, and LSS expression was induced as previously described (42). LSS activity was assessed as the conversion rate from $\left[{ }^{3} \mathrm{H}\right] 2,3$-oxidosqualene to lanosterol (42). Three separate assays were performed for each construct.

Aberrant splicing of Lss exon 4. Chromosomal DNA sequence from exon 4 to exon 5 of the Lss gene was PCR amplified from genomic DNA from normal SCRs, cataractous SCRs, and ACI rats using the Lss-19/Lss-2 primer pair (Figure 4C). PCR products were subcloned into the pEF6/V5-His-TOPO plasmid (Invitrogen Corp.), resulting in PEF-Lss ${ }^{A}-$ exon $4 / 5$ and $\mathrm{pEF}-L s s^{S}$-exon $4 / 5$. These minigene constructs were introduced into COS cells using Effectene Transfection Reagent (QIAGEN), and mRNA was isolated from the cells 2 days after transfection. This mRNA was reverse transcribed and PCR amplified using primers Lss-2 and Lss-19 that was 5' end-labeled with HEX dye.
PCR consisted of 18-32 cycles to give logarithmic phase amplification. The PCR products were run on an ABI PRISM 310 Genetic Analyzer (Applied Biosystems), and aberrant and normally spliced products were quantified by the GeneScan Analysis Software (version 2.1.1; Applied Biosystems).

FDFT1 activity assay. Rabbit polyclonal antibody was raised against FDFT1 protein expressed in E. coli and purified following previously described procedures (43) with minor modifications. Aliquot of $10 \mu \mathrm{g}$ liver microsomal protein were separated by SDS-polyacrylamide gel electrophoresis and transferred onto PVDF membranes. Membranes were then reacted with the antiserum and detected with ImmunoStar Reagents (Wako Pure Chemical Industries Ltd.) for chemical luminescence. Signal densities on X-ray films were determined using an ATTO densitograph 4.1 system (ATTO). The FDFT1 activity assay was performed on liver microsomal fractions basically as previously described (44).

Sterol measurement. Three rats were used per group. Blood samples were collected after overnight fasting, and ocular lenses and part of the liver and frontal cerebral cortex were removed and homogenized in distilled water. Total lipids were extracted by the standard Bligh-Dyer method. Total cholesterol content was measured by enzymatic methods (Determiner TC555; Kyowa Medex Co. Ltd.), and difference in mean cholesterol content was evaluated by 2 -tailed Student's $t$ test. For determination of sterol profile, squalene, 2,3-oxidosqualene, lanosterol, 7-dehydrocholesterol, and desmosterol were purchased from Sigma-Aldrich and used as standards in a gas-liquid chromatography using a GC-17A gas chromatograph (Shimadzu Corp.) equipped with a chemically bonded fused silica SPB-1 capillary column (Sigma-Aldrich). Two lenses from each rat were combined, and $100 \mu \mathrm{g} 5 \mathrm{a}$-cholestane was added as an internal calibration standard. Sterols were extracted with chloroform-methanol and quantified by gas-liquid chromatography.

Examination of embryos. Embryos were harvested from timed matings of cataractous SCR. Genotyping of embryos and adult rats was performed on DNA isolated from embryo samples or adult tail tips by PCR amplification using an Lss-11 and Lss-12 primer pair (Figure 3A) that could differentiate the presence $\left(L s s^{S}\right.$ allele) and absence ( $L s s^{l}$ allele) of a $13-b p$ sequence (Figure $8 \mathrm{~A}$ ). $\mathrm{PCR}$ products were run on $4 \% \mathrm{NuSieve}$ agarose gel. Embryos were fixed using buffered formalin, embedded in paraffin, and stained with hematoxylin and eosin.

\section{Acknowledgments}

This work was supported in part by a Grant-in-Aid for Scientific Research from the Japan Society for the Promotion of Science (17500286).

Received for publication December 12, 2003, and accepted in revised form November 22, 2005.

Address correspondence to: Masayuki Mori, Department of Aging Biology, Institute on Aging and Adaptation, Shinshu University Graduate School of Medicine, 3-1-1 Asahi, Matsumoto 390-8621, Japan. Phone: 81-263-37-2692; Fax: 81-263-37-3428; E-mail: masamori@sch.md.shinshu-u.ac.jp.
1. Tripathi, B.J., Tripathi, R.C., Borisuth, N.S., Dhaliwal, R., and Dhaliwal, D. 1991. Rodent models of congenital and hereditary cataract in man. Lens Eye Toxic. Res. 8:373-413.

2. Shumiya, S. 1995. Establishment of the hereditary cataract rat strains (SCR) and genetic analysis. $L a b$. Anim. Sci. 45:671-673.

3. Okano, T., Uga, S., Ishikawa, S., and Shumiya, S. 1993. Histopathological study of hereditary cataractous lenses in SCR strain rat. Exp. Eye Res. 57:567-576
4. Okano, T., Uga, S., Ishikawa, S., Hara, A., and Shumiya, S. 1999. Lens reconstruction after cataract in SCR rat. Jpn. J. Opthalmol. 43:363-367.

5. Hightower, K.R., and McCready, J.P. 1991. Effect of selenite on epithelium of cultured rabbit lens. Invest. Ophthalmol. Vis. Sci. 32:406-409.

6. Li, W.C., and Spector, A. 1996. Lens epithelial cell apoptosis is an early event in the development of UVB-induced cataract. Free Radic. Biol. Med. 20:301-311.

7. Takamura, Y., Kubo, E., Tsuzuki, S., and Akagi, Y.
2003. Apoptotic cell death in the lens epithelium of rat sugar cataract. Exp. Eye Res. 77:51-57.

8. Li, W.C., et al. 1995. Lens epithelial cell apoptosis appears to be a common cellular basis for noncongenital cataract development in humans and animals. J. Cell Biol. 130:169-181.

9. Inomata, M., Hayashi, M., Shumiya, S., Kawashima, S., and Ito, Y. 2001. Involvement of inducible nitric oxide synthase in cataract formation in Shumiya cataract rats (SCR). Curr. Eye Res. 23:307-311.

10. Inomata, M., et al. 1997. Evidence for the involve- 
ment of calpain in cataractogenesis in Shumiya cataract rat (SCR). Biochim. Biophys. Acta. 1362:11-23.

11. Inomata, M., Hayashi, M., Shumiya, S., Kawashima, S., and Ito, Y. 2000. Aminoguanidine-treatment results in the inhibition of lens opacification and calpain-mediated proteolysis in Shumiya cataract rats (SCR). J. Biochem. 128:771-776.

12. Zhang, H., et al. 2001. Peptidases play an important role in cataractogenesis: an immunohistochemical study on lenses derived from Shumiya cataract rats. Histochem. J. 33:511-521.

13. Cenedella, R.J., and Bierkamper, G.G. 1979. Mechanism of cataract production by 3-beta(2-diethyl aminoethoxy)andost-5-en-17-one hydrochloride, U18666A: an inhibitor of cholesterol biosynthesis. Exp. Eye Res. 28:673-688.

14. Cenedella, R.J. 1983. Source of cholesterol for the ocular lens, studied with U18666A: a cataract-producing inhibitor of lipid metabolism. Exp. Eye Res. 37:33-43.

15. Cenedella, R.J. 1985. Regional distribution of lipids and phospholipase A2 activity in normal and cataractous rat lens. Curr. Eye Res. 4:113-120.

16. Yadav, S., and Rawal, U.M. 1992. Cholesterol and lipid peroxidation in 3beta-(2-diethylaminoethoxy)androst-5-en-17-oneHCl (U18666A) induced cataractogenesis in rats. Indian J. Exp. Biol. 30:147-148.

17. Pyrah, I.T., et al. 2001. Toxicologic lesions associated with two related inhibitors of oxidosqualene cyclase in the dog and mouse. Toxicol. Pathol. 29:174-179.

18. Gong, X., et al. 1997. Disruption of alpha3 connexin gene leads to proteolysis and cataractogenesis in mice. Cell. 91:833-843.

19. Cheng, Q., Robinson, W.G., and Zigler, J.S., Jr. 2002. Geranylgeranyl pyrophosphate counteracts the cataractogenic effect of lovastatin on cultured rat lenses. Exp. Eye Res. 75:603-609.

20. Chen, H.W., Heininger, H.J., and Kandutsch, A.A. 1975. Relationship between sterol synthesis and DNA synthesis in phytohemagglutinin-stimulated mouse lymphocytes. Proc. Natl. Acad. Sci. U. S. A.
72:1950-1954.

21. Martinez-Botas, J., et al. 2001. Dose-dependent effects of lovastatin on cell cycle progression. Distinct requirement of cholesterol and non-sterol mevalonate derivatives. Biochim. Biophys. Acta. 1532:185-194

22. Suarez, Y., et al. 2005. Sterol stringency of proliferation and cell cycle progression in human cells. Biochim. Biophys. Acta. 1734:203-213.

23. Dietschy, J.M., and Turley, S.D. 2002. Control of cholesterol turnover in the mouse. J. Biol. Chem. 277:3801-3804.

24. Jollie, W.P. 1990. Development, morphology, and function of the yolk-sac placenta of laboratory rodents. Teratology. 41:361-381.

25. Porter, F.D. 2002. Malformation syndromes due to inborn errors of cholesterol synthesis. J. Clin. Invest. 110:715-724. doi:10.1172/JCI200216386.

26. Cendella, R.J. 1996. Cholesterol and cataracts. Surv. Ophthalmol. 40:320-337.

27. Berger, S., Smit, G.P., Schierbeek, H., Bijsterverd, K., and le Coultre, R. 1985. Mevalonic aciduria: an inborn error of cholesterol biosynthesis? Clin. Chim. Acta. 152:219-222.

28. Hoffman, G., et al. 1986. Mevalonic aciduria-an inborn error of cholesterol and nonsterol isoprene biosynthesis. N. Engl. J. Med. 314:1610-1614.

29. Hoffman, G.F., et al. 1993. Clinical and biochemical phenotype in 11 patients with mevalonic aciduria. Pediatrics. 91:915-921.

30. Cotlier, E., and Rice, P. 1971. Cataracts in the Smith-Lemli-Opitz syndrome. Am. J. Ophthalmol. 72:955-959.

31. Gold, J.D., and Pfaffenbach, D.D. 1975. Ocular abnormalities in the Smith-Lemli-Opitz syndrome. J. Pediatr. Ophthalmol. 12:228-234.

32. Kretzer, F.L., Hittner, H.M., and Mehta, R.S. 1981. Ocular manifestations of the Smith-Lemli-Opitz syndrome. Arch. Ophthalmol. 99:2000-2006.

33. Happle, R. 1979. X-linked dominant chondrodysplasia punctata. Review of literature and report of a case. Hum. Genet. 53:65-73.
34. Happle, R. 1995. X-linked dominant chondrodysplasia punctata/ichthyosis/cataract syndrome in males [letter]. Am. J. Med. Genet. 57:493.

35. Cenedella, R.J. 1982. Sterol synthesis by the ocular lens of the rat during postnatal development. J. Lipid Res. 23:619-626.

36. El-Sayed, G.N., and Cenedella, R.J. 1987. Relationship of cholesterolgenesis to DNA synthesis and proliferation by lens epithelial cells in culture. Exp. Eye Res. 45:443-451.

37. Schlienger, R.G., Haefeli, W.E., Jick, H., and Meier, C.R. 2001. Risk of cataracts in patients treated with statins. Arch. Intern. Med. 161:2021-2026.

38. Gerson, R.J., et al. 1990. On the etiology of subcapsular leticular opacities produced in dogs receiving HMG-CoA reductase inhibitors. Exp. Eye Res. 50:65-78.

39. Cenedella, R.J., et al. 2004. Direct perturbation of lens membrane structure may contribute to cataracts caused by U18666A, an oxidosqualene cyclase inhibitor. J. Lipid Res. 45:1232-1241.

40. Cenedella, R.J., Kuszak, J.R., Al-Ghoul, K.J., Qin, S., and Sexton, P.S. 2003. Discordant expression of the sterol pathway in lens underlies simvastatininduced cataracts in Chbb:Thom rats. J. Lipid Res. 44:198-211.

41. Abe, I., Bai, M., Xiao, X., and Prestwich, G.D. 1992. Affinity labeling of vertebrate oxidosqualene cyclases with a tritiated suicide substrate. Biochem. Biophys. Res. Commun. 17:32-38.

42. Abe, I., and Prestwich, G.D. 1995. Molecular cloning, characterization, and functional expression of rat oxidosqualene cyclase cDNA. Proc. Natl. Acad. Sci. U. S. A. 92:9274-9278.

43. Thompson, J.F., et al. 1998. Truncation of human squalene synthase yields active, crystallizable protein. Arch. Biochem. Biophys. 350:283-290.

44. Ishihara, T., Kakuta, H., Moritani, H., Ugawa, T., and Yanagisawa, I. 2004. Synthesis and biological evaluation of novel propylamine derivatives as orally active squalene synthase inhibitors. Bioorg. Med. Chem. 12:5899-5908. 